Sergey Androsov

\title{
DOMENICO TREZZINI E ALTRI MAESTRI TICINESI A PIETROBURGO E TALLINN ALL'INIZIO DEL SETTECENTO
}

L'attività dell'architetto e ingegnere oriundo del Canton Ticino Domenico Trezzini (Astano 1670 - San Pietroburgo 1734) è stata generalmente studiata da storici russi. Nel 1986 e nel 1988 sono state pubblicate due monografie in russo dedicate a Trezzini dal carattere divulgativo. Queste opere hanno anche favorito la pubblicazione di diversi documenti riguardanti la vita e l'opera dell'architetto. ${ }^{1}$

Un contributo ancor più importante è stata la mostra tenutasi nel Museo Cantonale dell'arte di Lugano intitolata "Domenico Trezzini e la costruzione di San Pietroburgo" (1994). ${ }^{2}$ I materiali provenienti dall'Archivio Statale Storico Russo, dall'Archivio Statale della Flotta marittima russa, dalla Biblioteca dell'Accademia delle Scienze, dal Museo statale Ermitage di Pietroburgo e dal Museo Statale Storico di Mosca, hanno presentato diversi aspetti della vita e dell'attività di Domenico Trezzini.

DOI: https://doi.org/10.12697/BJAH.2019.17.02

1 Irina I. Lisaevič, Domenico Trezzini (Leningrado: Iskusstvo, 1986); Yurij M. Ovsiannikov, Domenico Trezzini (Leningrado: Iskusstvo, 1988); Konstantin V. Malinovsky, Dominico Trezini (Sankt-Peterburg: Kriga, 2007).

2 Domenico Trezzini e la costruzione di San Pietroburgo. Catalogo della mostra a cura di Marco Franciolli, Manuela Kahn-Rossi (Firenze: Octavo, 1994). 
Allo stesso tempo, restano ancora delle lacune nella biografia dell'architetto ticinese. Infatti, non è chiaro come Trezzini sia capitato in Danimarca né perché egli si sia successivamente trasferito in Russia. Uno storico danese, Harald Langberg, ha sostenuto che Trezzini si era legato ad un altro maestro ticinese, Domenico Pelli, attivo in Danimarca dal 1688, come architetto delle fortificazioni e ingegnere. Langberg ha pubblicato anche i passaporti rilasciati per un viaggio da Copenhagen ad Arkanghelsk di un gruppo di ticinesi e italiani, dove figura anche il nome di "Domenicus Trezzini". ${ }^{3}$

È cosa nota che il contratto per Trezzini e per altri ticinesi fu concluso con il ministro russo in Danimarca, Andrej Petrovič Izmajlov, il quale non si distinse né per un particolare amore per l'arte, né per l'invio di altri maestri in Russia. Evidentemente l'invito ai ticinesi fu il risultato di un felice concorso di circostanze. Fondandoci su dati posteriori, possiamo ipotizzare una spiegazione per l'improvviso interesse artistico di Izmajlov.

Nel luglio del 1719, Giuseppe di Andrea Franchi - originario di Bergamo e assente dalla patria da più che dieci anni - era tornato a Venezia dalla Russia. Gli inquisitori di Stato veneziani, interessati a raccogliere informazioni sul lontano paese nordico, naturale alleato di Venezia nella Guerra contro l'Impero Ottomano, si affrettarono a interrogare Franchi sul suo viaggio e sulla sua posizione in Russia. Dalle risposte di Franchi risulta che egli lasciò l'Italia insieme ad alcuni nobili russi che avevano studiato a Venezia. Dopo un soggiorno di dieci mesi in Russia si era recato in Prussia e in Danimarca, dove aveva assolto alcuni incarichi affidatigli dallo zar Pietro I (Pietro il Grande). ${ }^{4}$

Queste notizie corrispondono pienamente con quanto ci è noto di Izamajlov. Dopo gli studi in Italia, egli aveva lasciato Venezia probabilmente alla fine del 1698, varcando il confine di Neuchausen (tra Riga e Pskov) il 3 marzo 1699, facendo ritorno in patria. ${ }^{5}$ In seguito, il 25 settembre 1700, Andrej Izmajlov ricevette l'ordine di partire come ambasciatore per la Danimarca, ma quasi contemporaneamente fu

3 Domenico Trezzini e la costruzione di San Pietroburgo, 219-221.

4 Sergey O. Androsov, "Džueppe Franki i ego svidetel'stvo o Rossii petrovskogo vremeni" ("Giuseppe Franchi e la sua testimonianza sulla Russia petrina"), Russica Romana, 5 (1998), 61.

5 Dmitrij J. Guzevič, Irina D. Gusevič, Velikoe posol'stvo. Rubež epoch, ili načalo puti. 16971698 (La Grande Ambasceria. Al confine di due epoche, o inizio del percorso) (San Pietroburgo: Dmitrij Bulanin, 2009), 442 . invitato in Prussia. Secondo i documenti russi, il suo passaggio in Danimarca va fatto risalire al 14 settembre 1702, mentre l'udienza d congedo a Copenhagen ebbe luogo il 5 dicembre 1707 ed egli tornò in Russia nell'agosto del $1708 .{ }^{6}$ Queste notizie coincidono perfettamente con gli spostamenti che Franchi comunicò agli inquisitori di Venezia. Da tutto questo si può dedurre che quest'ultimo si trovava al servizio di Izamajlov, probabilmente in qualità di consigliere o di aiutante, mentre più tardi Franchi divenne collaboratore del conte Savva Vladislavič. ${ }^{7}$ In questo caso si può supporre che avendo incontrato in Danimarca un gruppo di conterranei, o quasi conterranei dal Ticino, il bergamasco Giuseppe Franchi li abbia aiutati a entrare al servizio della Russia. Sembra che fu proprio Franchi l'ideatore del contratto concluso con il gruppo di capomastri, architetti e artigiani provenienti dal Canton Ticino e dall'Italia.

Nell'Archivio Statale Russo degli Atti Antichi (RGADA) è conservato un documento intitolato "Viaggio in Russia da Copenhagen dell'architetto Domenico Trezzini e dei mastri da muro stranieri", il quale contiene un'informazione interessante che non è stata valutata con sufficiente attenzione dai ricercatori che in passato si sono occupati della questione. ${ }^{8}$ Il passaggio più significativo del documento riguarda il contratto originale di Domenico Trezzini, redatto in italiano il 1 aprile 1703 con allegata la traduzione russa del tempo. Agli studiosi russi questo contratto era noto solo nella traduzione pubblicata nel 1911 da M. Korolkov. ${ }^{9}$ E proprio questo testo è stato riportato nel catalogo della mostra su Trezzini del 1994, dove venne tradotto dal russo in italiano..$^{10}$ Non è difficile intuire che la traduzione di una traduzione non corrisponde al testo originale del contratto che pubblichiamo in appendice al presente articolo.

La nave "Saugebon" sulla quale si erano imbarcati i ticinesi salpò da Copenhagen evidentemente alla fine del mese di maggio del 1703,

6 Nikolai N. Bantyš-Kamenskij, Obzor vnesnich snošenij Rossii (po 1800 god) (Rassegna dei rapporti esterni della Russia (fino al 1800)), I (Mosca, 1894), 7.

7 Sulla biografia di Franchi vedi: Androsov, "Džueppe Franki i ego svidetel'stvo o Rossii petrovskogo vremeni"

8 Questa busta è usata da Yurij M. Ovsiannikov, ma in maniera assai succinta (Ovsiannikov, Domenico Trezzini, 16-18, 20-22).

9 M. Korolkov, "Architekty Treziny" ("Gli architetti Trezzini"), Starye gody (San Pietroburgo, aprile 1911), 18.

10 Domenico Trezzini e la costruzione di San Pietroburgo, 220, 221. 
perché sia il passaporto di Trezzini, sia il nullaosta per la partenza della nave per Arkanghelsk recano la data del 26 maggio. ${ }^{11}$ Nella seconda metà di luglio i viaggiatori giunsero ad Arkanghelsk e dopo furono spediti per Vologda e Mosca. Esiste una segnalazione del voevoda Vasilij Rzevskij in data 27 luglio dalla quale si evince che chiesero del denaro "per le spese di sostentamento affrontate nel viaggio per nave e per il viaggio stesso" e il voevoda diede 10 rubli a ciascuno di loro, compreso "Il maestro ingegnere Domedico Trezy [Domenico Trezzini]". ${ }^{12}$ Arrivato a Mosca il 22 agosto 1703, Trezzini rifiutò il denaro offertogli ritenendolo insufficiente: “...e in base a quella disposizione le persone suddette l'ingegnere Dominik Trezy (10) rubli non ne prese, ma dice che egli già prima del suo arrivo a Mosca prese in prestito per nutrirsi molti danari e non piccolo numero di quei debiti deve pagare, ma anche nutrirsi dovrà per non pochi giorni...".13 Il risultato fu che nel mese di ottobre fu concessa a Trezzini, secondo l'accordo "oltre alla somma già percepita per il propriosostentamento, una nuova somma di denaro per l'ammontare complessivo di 20 rubli". ${ }^{14}$ In quella occasione, Trezzini fornì le seguenti informazioni di sé: "Ha studiato il mestiere di architetto e di ingegnere in Italia e da lì è partito per Copenhagen dove ha vissuto per 4 anni e realizzato molte fortificazioni e altre decorazioni di case con grande maestria, non fu mai al servizio di nessuno, in nessun luogo e perciò non possiede lettere di referenza". ${ }^{15}$ Va ancora una volta rilevato che Trezzini andò in Russia prima di tutto in qualità di ingegnere e specialista di fortificazioni, anche se riguardo a questa professione egli era considerato secondo nella lista degli

11 Harald Langberg, "Domenico Trezzini e San Pietroburgo visti da Copenhagen", Domenico Trezzini e la costruzione di San Pietroburgo, 220, 221. Invece, Yurij M. Ovsiannikov pensa che la nave salpò alla fine di giugno (Ovsiannikov, Domenico Trezzini, 19).

12 Archivio Statale Russo per gli Atti antichi [Rossiiskii gosudarstvennyi arkhiv drevnikh 15 (“Майстер инженерской Домедик Tрецы" - Maister inženierskoj Domenik Trecy - Mastro ingeniere Domenik Trezi).

13 Ibidem, f. 31 verso (“...и по тому указу из вышеписанных людей инженер Доминик Трецы (10) рублев не принял, а говорит, что уже он по приезде своем к Москве издержа. себе на прокормление занимая из долгу многие денги, и тем де малым числом не токмо ему з долгами оплатитца, но и кормитца будет малые дни...”).

14 Ibidem, f. 32 (“....указной дачи на прокормление к прежним дачам в зачет дватцать рублев...").

15 Ibidem, f. 43 (“А учился он архитектурской работе и инженерству во Италии и оттуда приехав жил в Копенгагене 4 года и делал многие фортификации и иные узорочные нет”). Il documento è citato da Yurij M. Ovsiannokov (Ovsiannikov, Domenico Trezzini, 20). specialisti, preceduto dal "Colonello Marco Emson, ingegnere con persone e possidente". ${ }^{16}$ Harald Langberg ha stabilito che si trattava del danese Marcus Heinsohn, esperto ingegnere militare, il quale aveva ricevuto dal re Federico IV l'incarico di fornire aiuto agli alleati russi. Secondo Langberg Heinsohn, lavorò in Russia almeno fino al 1707 e probabilmente tornò in patria l'anno successivo. ${ }^{17}$

Altretanto interessanti sono le notizie sugli altri mastri di origine ticinese che accompagnarono Trezzini durante il viaggio. L'ipotesi più circostanziata sulla loro attività a Mosca è stata avanzata da $\mathrm{T}$. A. Gatova. Basandosi sulle informazioni raccolte nella prima metà del XIX secolo da A. F. Malinovskij riguardo alla partecipazione dei "mastri intagliatori italiani" ai lavori per la chiesa estiva de monastero Bogojavlenskij, per la chiesa dell'Arcangelo Gabriele (la cosiddetta "torre Mensikov" a Mosca e per la Chiesa del Segno della Madre di Dio (Znamenia) presso Dubrovicy, la studiosa attribuiva loro la realizzazione di composizioni decorative e figurative in stucco tuttora conservate in quegli edifici religiosi. T. A. Gatova ha ipotizzato che questi artisti provenienti dalla Svizzera italiana avessero costituito un atelier che si occupò anche di fornire dei lavori in stucco a Mosca e nei dintorni, probabilmente tra $1703-1707 .{ }^{18} \mathrm{La}$ tesi di T. A. Gatova, sebbene fondata su fonti indirette piuttosto che su documenti d'archivio, è stata ritenuta attendibile dalla maggior parte degli studiosi che vennero dopo. Allo stesso tempo le critiche avanzate nei confronti di questa tesi furono lasciate cadere. Tuttavia, già nel 1985 A. Mikhajlov aveva rivelato come i mastri giunti con Trezzini non si definissero scultori, intagliatori o stuccatori (tranne un'eccezione), ma solo "mastri di fabbriche e fortificazioni". È pertanto poco probabile che essi fossero in grado di realizzare rilievi ed altre decorazioni in stucco. ${ }^{19}$ Più tardi Yu. Ovsiannikov asserì che "i mastri di batimenti" ticinesi assunti in Danimarca e chiamati a lavorare alla costruzione dell'Arsenale del Cremlino di Mosca, non erano in

16 RGADA, fondo 150, anno 1703, busta 12, f. 15 (“Подполковник инженер Марко Эмсон с людми и вещми").

17 Langberg, "Domenico Trezzini e San Pietroburgo visti da Copenhagen", 221, 222.

18 T. A. Gatova, "Iz istorii dekorativnoj skul'ptury Moskvy nacala XVIII v." ("Dalla storia Materialy i issledovanija (L'arte russa del XVIII secolo. Materiali e studi) (Mosca, 1973), 31-43.

19 A. Mikhajlov, "Podmoklovskaja rotonda i klassiceskie vejanija v iskusstve petrovskogo vremeni" ("La rotunda di Podmokloje e le correnti classiche nell'arte dell'epoca di Pietro"), skusstvo, 9 (1985), 70. 
grado di lavorare senza la supervisione di un architetto e per questo furono presto mandati via dalla Russia. ${ }^{20}$

Nei documenti che si riferiscono all'ingresso di Trezzini in Russia si può infatti conoscere anche il successivo destino dei suoi compagni di viaggio ticinesi. Nel febbraio del 1704 "per ordine di Sua Maestà Imperiale gli stranieri sopramenzionati sono stati inviati dal dicastero per gli Affari esteri all'Armeria per occuparsi dei lavori per lo zekaus [arsenale - S. A.]. Ma gli stranieri sopramenzionati sono stati esclusi dai lavori per zekaus, poiché hanno detto di non poter lavorare senza la direzione di un architetto e di un ingegnere capo, il quale sarebbe dovuto arrivare, ma è poi stato inviato a Novgorod e San Pietroburgo al servizio di Sua Maestà e non possono rimanere a lavorare in quelle condizioni perché non s'intendono di architettura, per cui è necessaria la guida di un maestro appositamente nominato, che sia preposto a tale opera di zekaus". ${ }^{21}$ Questa testimonianza non solo chiarisce i rapporti operativi tra Trezzini e il gruppo di mastri che lo accompagnavano, ma offre un fondamento per la datazione della partenza dell'architetto per San Pietroburgo, che evidentemente avvenne nella prima metà di febbraio del 1704. E nell'agosto Trezzini era già a Narva, recentemente conquistata dagli svedesi. Tra le prime costruzioni dell'architetto ricordiamo la Porta della città, probabilmente massiccia e pesante, decorata con una statua dell'apostolo Paolo, ma oggi non più esistente.

La sfortuna degli accompagnatori ticinesi fu un'altra. Il rifiuto di lavorare senza la guida di un architetto provocò l'irritazione di Pietro I. Nell'aprile $1704 \mathrm{fu}$ ordinato “...di congedare i capomastri italiani, con lo stipendio di 2 mesi e con il permesso di passare per la città di Arkanghelsk per poi imbarcarsi per mare; se invece desiderano restare a vivere in Russia, sia concessa loro l'autorizzazione". ${ }^{22} \mathrm{Ai}$ ticinesi furono assegnati i mezzi necessari per il viaggio da Mosca ad Arkanghelsk e furono dati i soldi che spettavano loro. Per questa

20 Ovsiannikov, Domenico Trezzini, 22

21 RGADA, fondo 150 , anno 1703, busta 12, ff. 46 verso, 47 verso (“...по указу Великого Государя те вышеименованные иноземцы к цекаузному делу неприняты; сказали что им ныне послан на его Велишиго архитектора мастера и инженера, которой был в приезде что они архитектуры не знают, и у того дела им самим мастерам быт немочно, разве под . 22 Ibidem, f. 47,47 verso (“...каменного дела мастеров италианцев дав им пасы, и жалованья на 2 месяца отпустить велено чрез город Архангелской за море, а ест ли похотят жить доброволно, дат им волю"). ragione si può supporre che essi lasciarono allora la Russia, sebbene non si possa escludere la possibilità che uno o due di loro avessero deciso di rimanere a lavorare in Russia a proprio rischio. Occorre fare un'ulteriore precisazione riguardo ai mastri giunti con Trezzini. Fino ad ora gli autori russi hanno utilizzato la trascrizione dei nomi in base ai documenti russi del tempo, che quasi sempre hanno distorto i nomi originali. Tuttavia esiste un documento dove i nomi dei mastri ticinesi sono presenti nella forma latina insieme alle loro professioni: “Dominicus Rusca pavimentarius et murarius, Iohannes Maria Fontana murarius, Carolus Ferrare murarius, Galeazzo Quadro murarius, Iohannes Maria Fontana murarius". ${ }^{23}$ Un elenco simile è stato pubblicato anche da H. Langberg. ${ }^{24} \mathrm{Si}$ noti la presenza di due omonimi Fontana, forse parenti. I loro nomi s'incontrano in questa forma in una serie di documenti, il che non si può spiegare come un semplice errore dello scrivano. Recentemente M. Nikolaeva ha pubblicato le firme di due maestri e ha ipotizzato che il secondo Fontana, firmato come Jean Marie Fontaine, era d'origine francese. ${ }^{25} \mathrm{Il}$ documento ha contribuito a creare la leggenda che uno dei Giovanni Maria Fontana non era ripartito dalla Russia, ma aveva lavorato per qualche tempo ancora a San Pietroburgo e fu l'architetto del Palazzo di Aleksandr Mensikov. In realtà esisteva ancora un altro architetto con il cognome Fontana, Francesco Fontana, probabilmente attivo a Mosca tra il 1706 e il 1708 e a Pietroburgo tra 1710 e 1713, il quale fu uno dei realizzatori del Palazzo di Menšikov. ${ }^{26}$

Va rilevato che sull'esilio dei ticinesi i materiali russi coincidono pienamente con quelli svizzeri. Secondo la lettera di Carlo Francesco Lavezzari, inviata al cugino Giuseppe Martinola a Mendrisio, spedita da Mosca nel 1706, Carlo Ferrario da Cadempino e Galeazzo Quadro

23 RGADA, f. 21 verso

24 Langberg, "Domenico Trezzini e San Pietroburgo visti da Copenhagen", 220 ("Iohannes Maria Fontana, Carolus Ferrun, Galeazzus Cuadro, Iohannes Maria Fontana").

25 M. V. Nikolaeva, "K voprosu o vyezde inostrannych masterov stroitel'nych i khudozestvennych professij v Rossiju pri Petre I po materialam RGADA" ("A proposito dell'entrata Gosudarstremogo E

26 Konstantin V. Malinovskij, "Arkhitektor A. D. Menšikova Francesco Fontana" ("Architetto di A.D. Menšikov Francesco Fontana"), Petrovskoe vremja v licach-2003 (San Pietroburgo, 2003), 75-80. Indicando i documenti sulle attività di Francesco Fontana a Mosca e a Pietroburgo l'autore ha tentato anche di riferire a Francesco Fontana diverse notizie in cui si tratta di un certo "architetto italiano". Oltre a Trezzini ha lavorato come architetto come vediamo più tardi, anche Giovanni Francesco Rossi. 
da Cassina d'Agno furono cacciati da Mosca "non essendo stati capaci di fare nulla". ${ }^{27}$ Questa testimonianza certamente si riferisce agli eventi del 1704 e ai maestri ticinesi di cui abbiamo appena parlato.

Bernardo Scala e Pietro Antonio Gielpi , arrivati da Copenhagen con Trezzini, non conclusero un contratto ufficiale con Izmailov. Abbiamo un interesse particolare per il secondo maestro, perché soltanto Gielpi era uno stuccatore e, come vedremo, proprio lui lavorò tempo dopo in Russia. Nel documento russo è chiamato Petr Gelpij con la segnalazione che era "arrivato a Mosca su invito dell'ambasciatore per incarichi edilizi e per risiedervi per il tempo desiderato, vivendo del proprio lavoro, perché a lui l'ambasciatore non aveva promesso denaro alcuno...". ${ }^{28}$ Non fu stipulato un accordo neppure con Bernardo Scala, il quale sembra fosse arrivato all'ultimo momento: "essere stato ingaggiato dall'ambasciatore mentre la nave stava per salpare e con la promessa di dargli quanto avrebbe dato agli altri compagni". ${ }^{29}$

A noi pare che le notizie raccolte contengano informazioni sufficienti per formulare alcune ipotesi. T. A. Gatova molto probabilmente si sbagliò nel riconoscere nel gruppo di mastri degli artisti capaci di realizzare rilievi in stucco all'interno delle chiese moscovite. Tuttavia, l'autrice era nel giusto quando sostenne che furono proprio i ticinesi, viaggiando per tutta Europa, ad occuparsi di questi lavori. Insistendo sulla paternità dei mastri svizzeri (Ticinesi) dell'intera serie di decorazioni a stucco delle chiese di Mosca, la studiosa proponeva come uno degli argomenti a sostegno della propria tesi la mancanza a Mosca in quel periodo di un'altra bottega in grado di eseguire tali opere. ${ }^{30} \mathrm{Ma}$ in realtà una bottega simile esisteva. Ne era a capo Giovanni Francesco di Cristoforo Rossi, giunto in Russia nel 1698, e forse ancor prima. È possibile che proprio Rossi, originario di Sessa, come indica Aldo Crivelli, ${ }^{31}$

27 Giuseppe Martinola, Lettere dai paesi Transalpini degli artisti di Meride e dei villagg vicini (XVII-XVIII) (Bellinzona: Ed. dello Stato, 1963), 21.

28 RGADA, fondo 150, anni 1703, busta 12, f. 21 verso (“...приехал к Москве по посолскому призыву для палатного дела жить на Москве сколко пожелает и кормитца тем мастерством, и ничего ему того посол денег не обещал...”).

29 Ibidem, f. 33 (“... посол ево принял при отпуске караблей и обещал ему давать против ево братьи").

30 Gatova, "Iz istorii dekorativnoj skul'ptury Moskvy nacala XVIII v.", 39

31 Aldo Crivelli, Artisti ticinesi in Russia (Locarno: Unione di Banche Svizzere, 1966), 53. fosse l'unico artista nella Russia del tempo specializzato in lavori in stucco. Purtroppo, fino ad oggi non sono stati trovati document relativi al suo arrivo in Russia. In seguito, egli stesso diede notizie contraddittorie in merito a questo argomento. Così nel gennaio del 1718 , nel presentare la richiesta di ritorno in patria, fu lo stesso Rossi a indicare come durata del suo servizio in Russia, 21 anni (cioè sembra che fosse arrivato nel 1696): “Ho servito Sua Maestà in opera di stucco e di architettura con tutta la mia fedeltà per 21 anni". ${ }^{32}$ In un altro documento dello stesso periodo egli riferiva di essere arrivato in Russia nel $1698 .^{33}$

Informazioni molto interessanti sull'attività di Rossi si trovano nella corrispondenza dei membri dell'Ordine dei Gesuiti a Mosca, pubblicate in traduzione russa nel 1904. Il 28 aprile del 1707 il gesuita Francesco Emiliano riferiva del successo della costruzione della chiesa cattolica della Santa Trinità a Mosca: "L'edificazione della nostra chiesa in pietra viene ora completata al suo interno con lavori di rifinitura i quali, iniziati dall'altare maggiore, ad eccezione delle icone della Santissima Trinità, quale sarà eseguito in gesso [stucco - S. A.] e ospiterà anche le statue del santo padre [Sant'Ignazio - S A.] e del santo apostolo delle Indie [San Francesco Saverio - S. A.]. Il costruttore desiderava aggiungere queste statue come segno della propria gratitudine perché la sua consorte italiana, che rischiava di morire di parto, riuscì a dare alla luce il suo bambino non appena le fu portata l'icona del santo padre e da quel momento si trova insieme col bambino di ottima salute". ${ }^{34}$

Già nel 1998 Vera Kovrigina ha identificato il costruttore della chiesa e l'autore di due statue in stucco in Giovanni Francesco Rossi e il bambino nato nel 1704 in Ignazio (Ignazio Lodovico) Rossi, anch' egli noto in Russia come stuccatore e architetto, chiamato così in onore del fondatore dell'Ordine dei gesuiti. ${ }^{35}$ Si può concludere che Giovanni Francesco Rossi non fu solo l'autore del progetto della prima chiesa

32 Archivio Storico Statale russo [Rossijskij Gosudarstvennyj Istoriceskij Archiv, RGIA], fondo 467 , inv. 4, busta 118 , f. 125 (“Служил я вашему Величеству при штукаторном и архитектурных делах со всею верностию дватцат один год...”).

33 Ibidem, f. 127

34 Pis'ma i donesenia iezuitov o Rossii konca XVII i načala XVIII veka (Lettere e rapporti dei gesuiti sulla Russia di fine XVII e inizio XVIII secolo) (San Pietroburgo, 1904), 159.

35 Vera A. Kovrigina, Nemeckaja sloboda Moskvy i ee obitateli v konce 17 - pervoj četvert 18 veka (Il quartiere tedesco di Mosca e $i$ suoi abitanti alla fine del XVII e nel primo quarto del XVIII secolo) (Mosca: Archeografičeskij Cent, 1998), 172. 


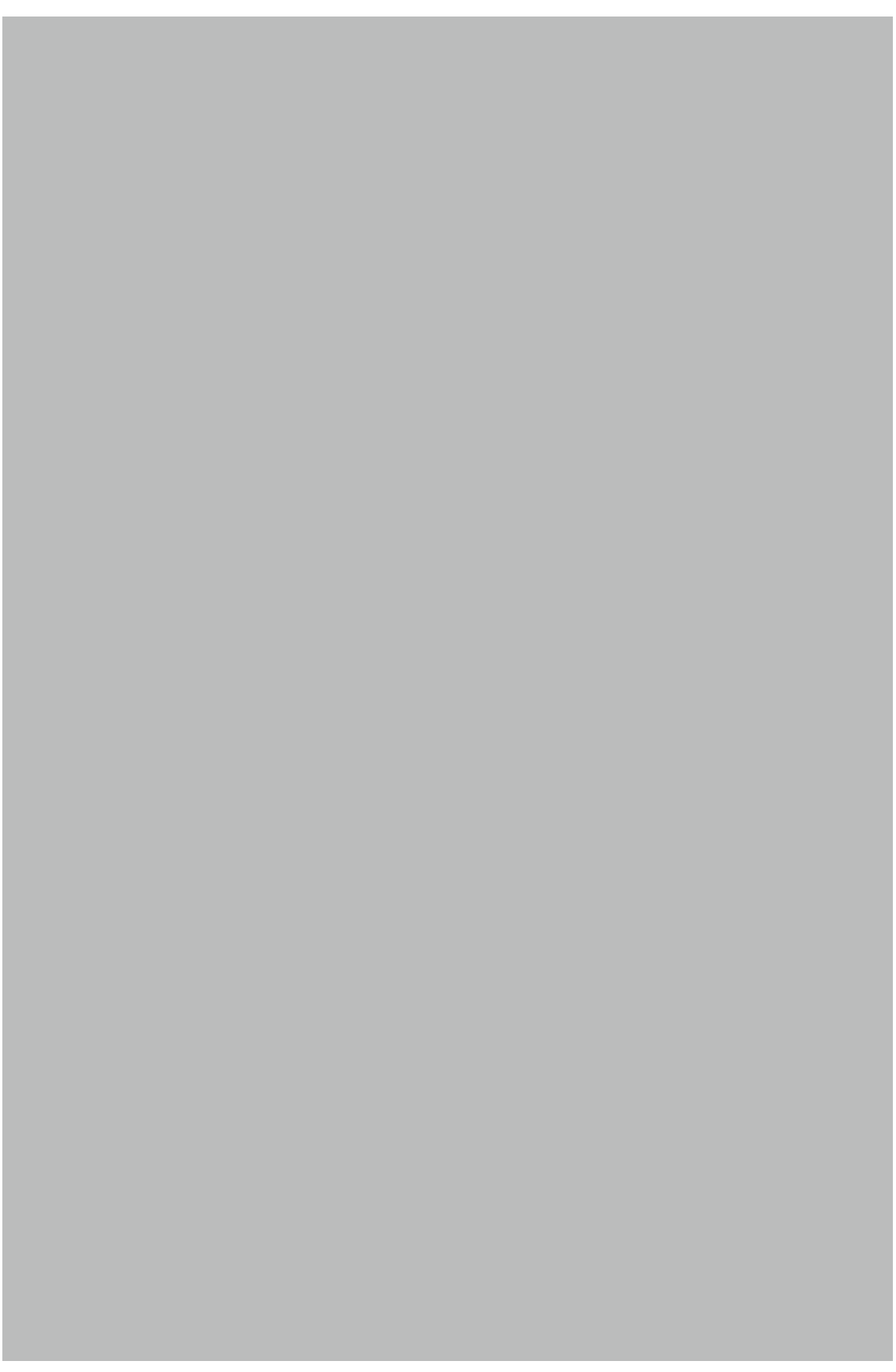

Ill. 1. Giovanni Francesco Rossi (?). Crocifissione. Dubrovicy, Chiesa del Segno della Madre di Dio.

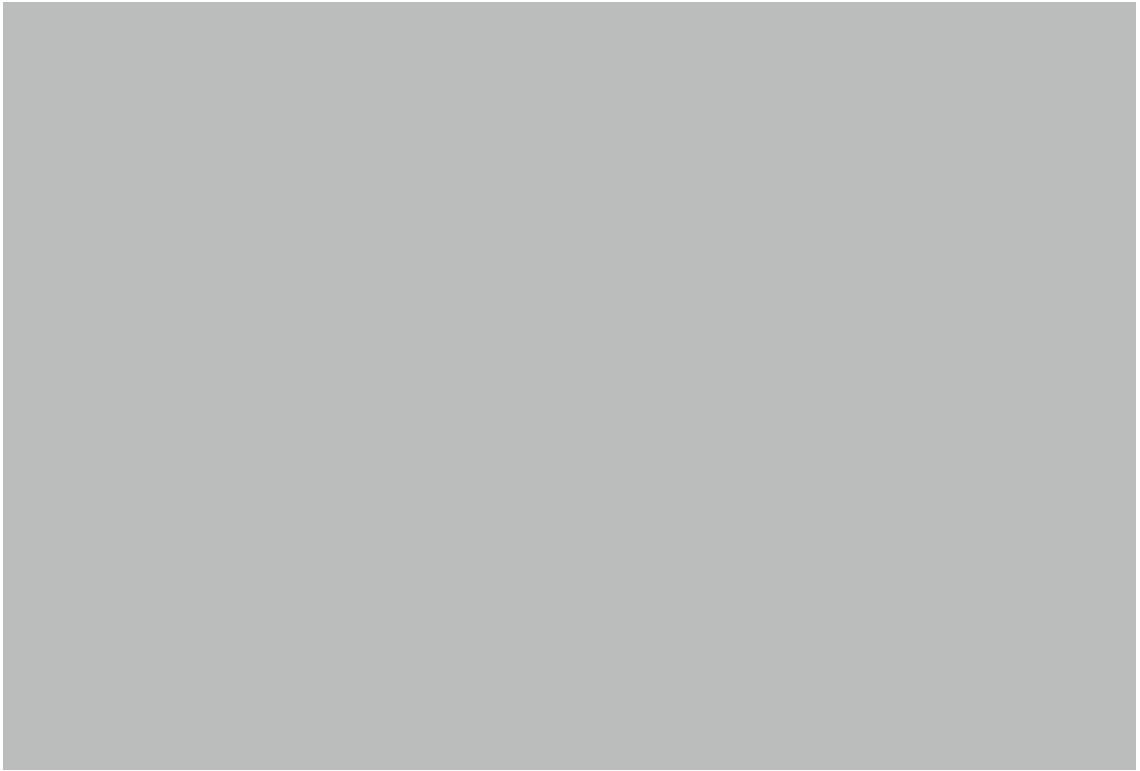

Ill. 2. Giovanni Francesco Rossi (?). Deposizione. Dubrovicy, Chiesa del Segno della Madre di Dio.

cattolica di Mosca, ma anche che eseguì due statue in stucco per l'altare maggiore della chiesa. Non ci sono dubbi riguardo al fatto che Rossi in quel momento dirigesse una bottega che si occupava di lavori in stucco. A nostro parere, è possibile attribuire l'esecuzione delle decorazioni in stucco delle chiese di Mosca e della chiesa del Segno della madre di Dio (Znamenija), presso Dubrovicy, il monumento più importante dell'arte della fine del Seicento a Mosca, a Giovanni Francesco Rossi e alla sua bottega.

È possibile reperire ulteriori informazioni sulla bottega di Rossi in un documento più tardo, datato dicembre 1717 , dal quale si evince che vi lavoravano non solo italiani, ma anche allievi russi: "Secondo il sopraindicato elenco firmato del maestro stuccatore Giovanni Rossi ha detto che nella sua permanenza al servizio di Sua Maestà Imperiale, per l'arte dello stucco, egli insegnò non solo al maestro straniero Antonio Kuadrij, ma anche ai russi Stepan Ivanov, Timofej Michailov, Semen Borisov, Ivan Kornilov, Petr Efremov, Vasilij Nikitin Vasilij Dmitreev, Leontij Timofeev, Petr Michailov e ancora ad un'altra 


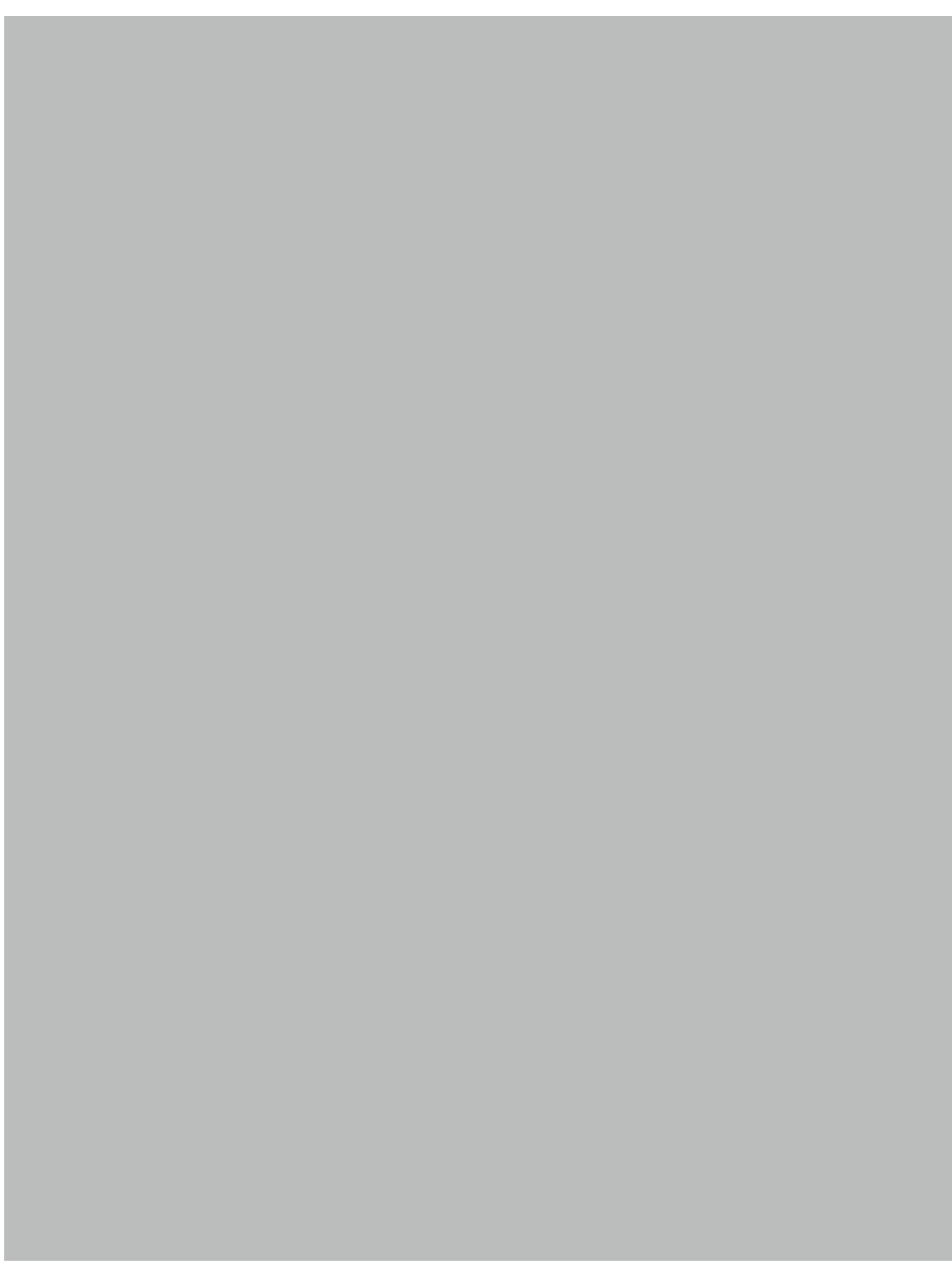

Ill. 3. Giovanni Francesco Rossi (?). Dio Padre con I angeli. Dubrovicy, Chiesa del Segno della Madre di Dio. decina di persone, di cui egli non rammentava il nome, i quali sanno ben lavorare come stuccatori, si trovano a Mosca e a San Pietroburgo e sono abili in tale arte più dei sopracitati, essendo tutti suoi allievi". ${ }^{36}$ Sembra evidente che Rossi avesse tutte le ragioni per essere orgoglioso dei propri successi nella formazione di allievi russi, incoraggiati specialmente da Pietro I.

Nell'elenco degli allievi di Rossi spicca il nome di Antonio Quadri (Quadrio), il cui cognome stesso rivela l'origine ticinese. Non bisogna confonderlo con Galeazzo Quadri, il quale, come abbiamo visto in precedenza, aveva lasciato la Russia nel 1704. Nella seconda metà degli anni 1710 il nome di Antonio Quadri è ricordato più volte nei documenti della Cancelleria per l'edilizia perché legato ai lavori in Peterhof. Egli, per esempio, firmò un contratto per i lavori in stucco il 1 agosto $1719 .{ }^{37}$ Qualche mesi dopo, nel dicembre del 1719 egli fece domanda di licenza dal servizio russo per questioni familiari, scrivendo "Io sono servitore di Sua Maestà zariana per i lavori di stuccatura dall'anno 700 fino ad oggi e nei precedenti anni ho lavorato per Sua Maestà a Peterhof, assolvendo la mia attività di stuccatore con sincera coscienziosità e portando a compimento numerose opere...". ${ }^{38}$ Per argomentare la necessità della partenza, Quadri fece riferimento alla notizia della morte dei genitori e quindi alla necessità di tornare in patria per le pratiche relative all'eredità. Purtroppo, egli non indica neanche una volta né il suo luogo di provenienza, né dove risiedevano i suoi genitori. La richiesta di Antonio Quadri fu accolta alla fine del 1719 ed egli partì alla volta della sua patria, dopodiché tornò in Russia. Nella primavera del 1721 Antonio Quadri visitò Riga per controllare le cave di pietra locale. ${ }^{39}$ Nella seconda metà del 1723 lavorò anche a Tallinn, dove eseguì in stucco la decorazione della

36 RGIA, fondo 467, inv. 4, busta 118, f. 126 (“... по вышеписанному подписанию штукаторного дела мастера Иоанн Росии сказал а штукаторному художеству в бытность ево у дел Его Царского Величеста изуи н за сущего мастера иноземца Антония Квадрия Петра Ефремова, Василия Никитина, Василия Диитреева, Леонтья Тимофеева, Петра Михайлова и еше ко оным человек з десят а имян им не помнит, которые штукаторную работу умеют работат добрым мастерством также которые обретаются в Москве и в Санкт Питер Бурхе сверх вышеписанных искусны штукаторному художеству все его ученики").

37 Ibidem, busta 806, f. 11.

38 Ibidem, busta 118, f. 126 (“.... служу я всенижайший вашему царскому величеству с 700-го года в делех штукаторныя работы даже и доныне как и в нынешних прешедших летех работал вашему величеству в петер гофе повеленную мне штукаторную работу со всеусердным моим прилежанием которая много работа преуспела ко окончанию...”).

39 Ibidem, fondo 470 , inv. 1, busta 21, ff. 36 recto, 56 recto. 


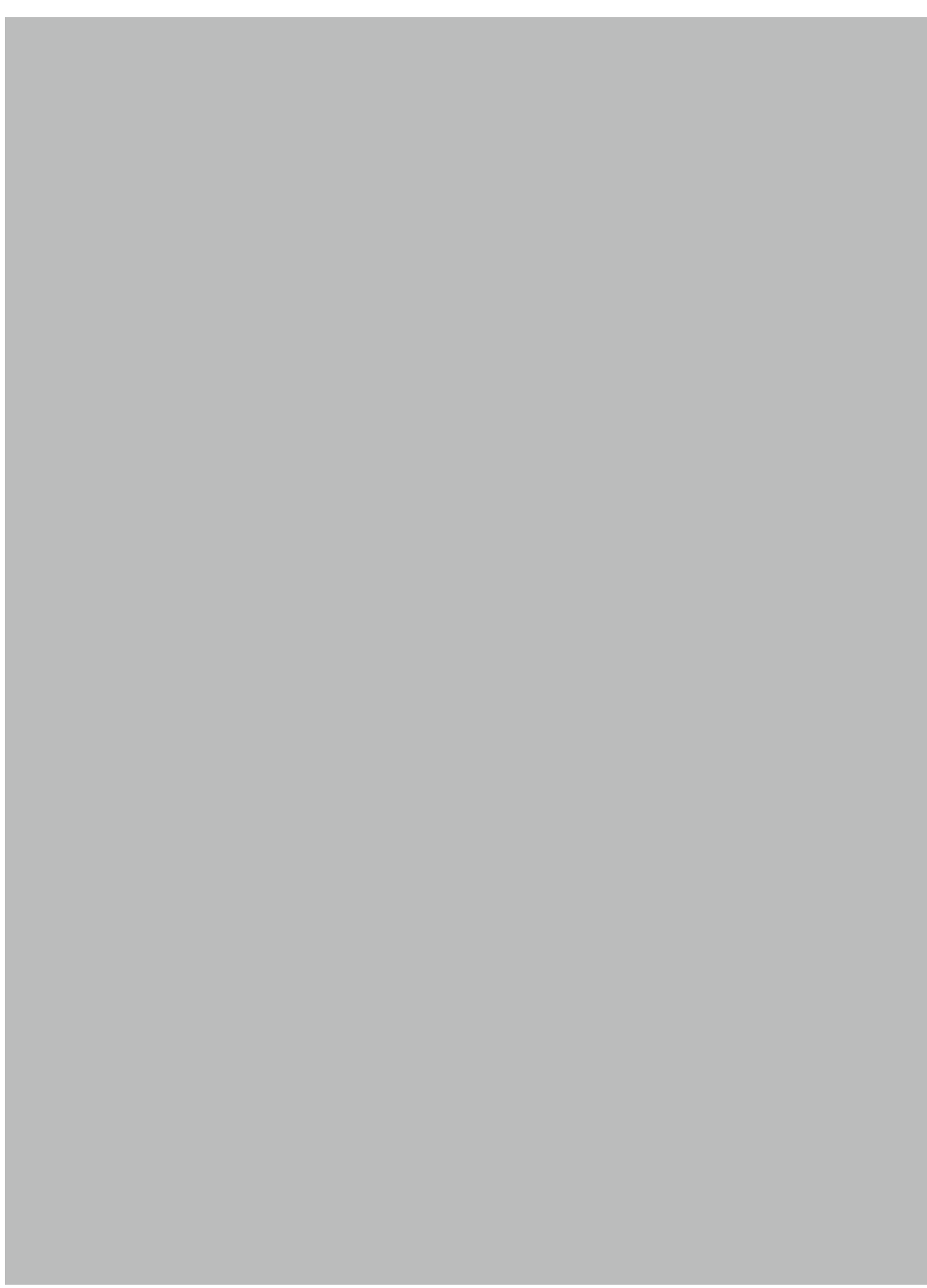

Ill. 4. Antonio Quadri (?). Dettaglio della sala principale del Palazzo di Kadriorg.

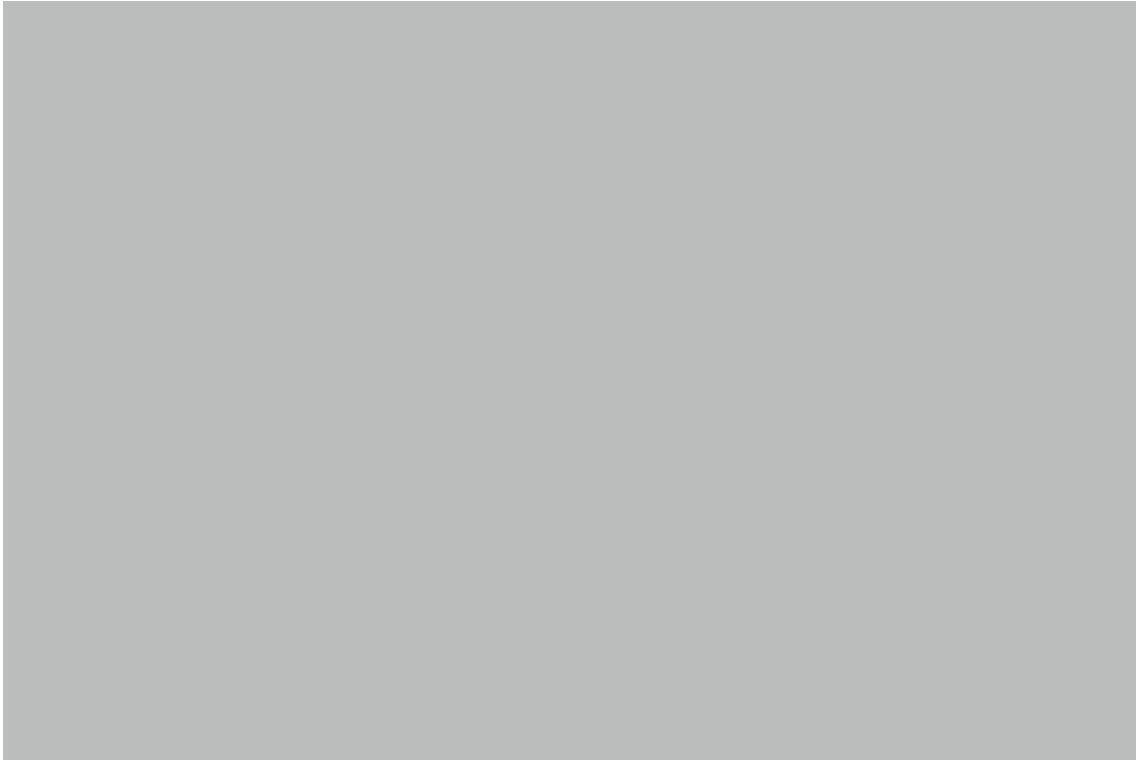

IIl. 5. Antonio Quadri (?). Dettaglio della sala principale del Palazzo di Kadriorg.

sala principale del Palazzo di Kadriorg con le figure degli angeli e le conchiglie. ${ }^{40}$ Il 26 dicembre 1723 spedì una lettera da Tallinn, nella quale chiese il permesso di ritornare a Pietroburgo. ${ }^{41}$ All'inizio dell'anno 1725, Quadri ricevette l'incarico di visitare nuovamente la città di Riga al fine di organizzare i lavori nelle cave della pietra locale destinata per Pietroburgo e Tallinn. Dalla discussione tenuta nella Cancelleria il 11 marzo si evince che Quadri fu molto occupato a Strelnja, Peterhof e nella chiesa di SS. Pietro e Paolo a Pietroburgo. Perciò la Cancelleria ha deciso spedire insieme con Quadri un allievo, Ivan Sevrin, per continuare i lavori dopo della partenza del suo $40 \quad$ I documenti sui lavori di Antonio Quadri nel Palazzo di Kadriorg sono pubblicati in lingua estone da Jüri Kuuskemaa: Juta Keevallik, "Kadrioru kroonika 1718-1727", Artiklite Kogumik 1976 (Tallinn: Eesti NSV Riiklik Kunstimuuseum, 1977), 64, 74-76. Ringrazio la gentilmente fornito questa informazione. Vedi anche: Kadriorg Palace: Kadriorg Art Museum (Tallinn: Kadriorg Art Museum, 2001), 19.

41 RGIA, fondo 467, inv. 2, busta 37b, f. 699. 


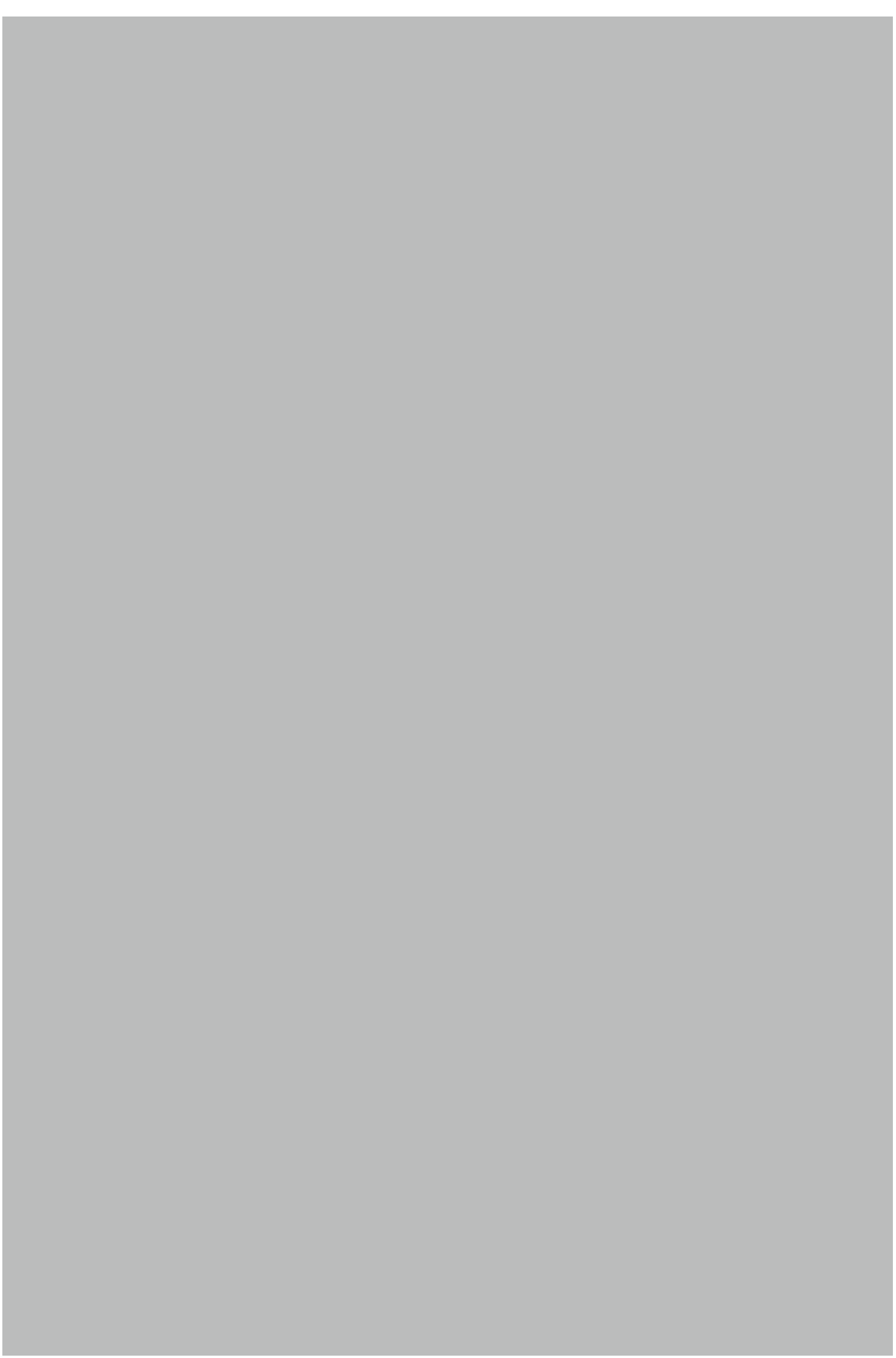

Ill. 6. Antonio Quadri (?). Dettaglio della sala principale del Palazzo di Kadriorg.

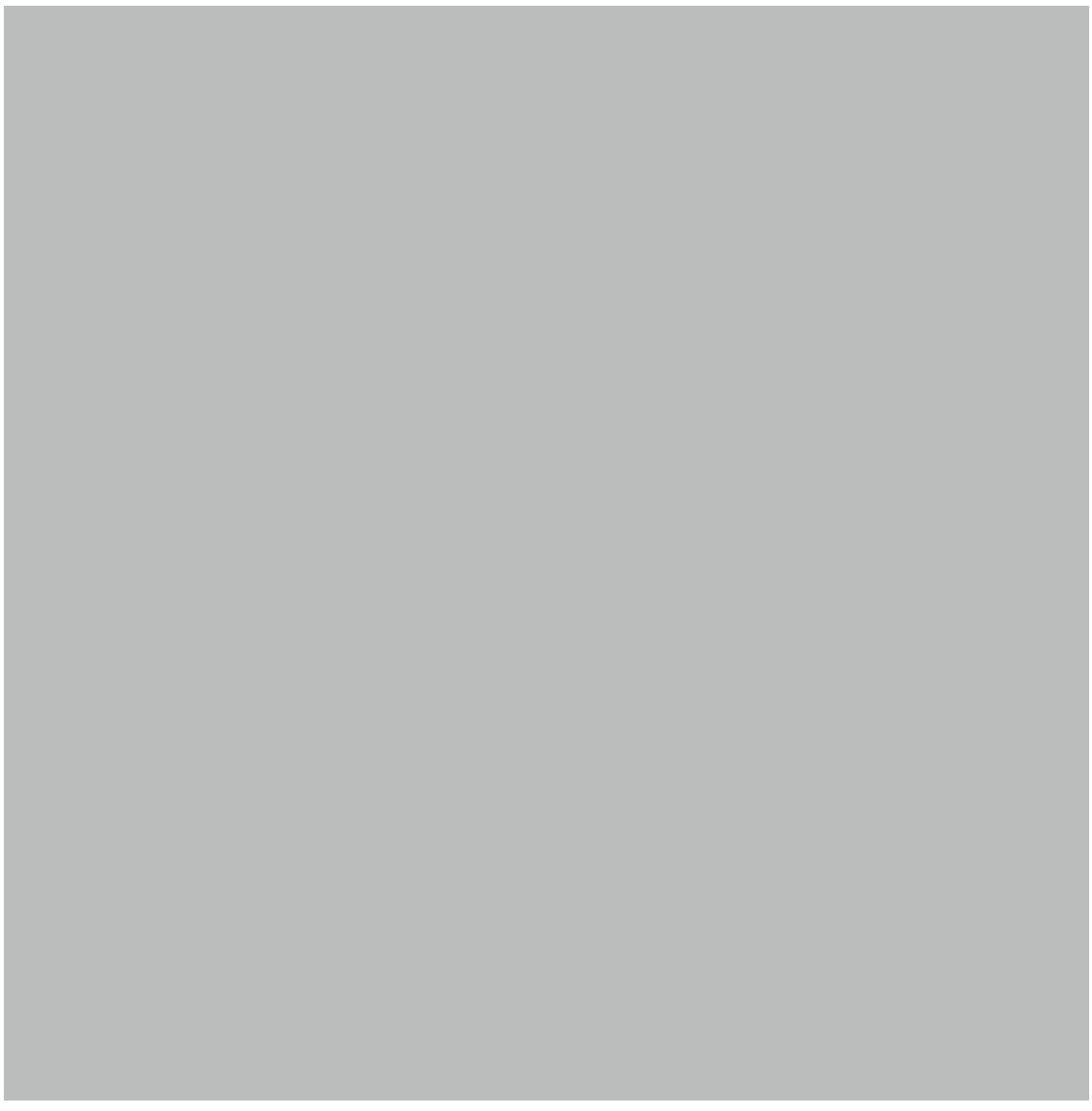

Ill. 7. Antonio Quadri (?). Dettaglio della sala principale del Palazzo di Kadriorg.

maestro. ${ }^{42}$ Nel novembre 1727 Quadri lavorò a Peterhof, dove eseguì sette rilievi in stucco per il palazzo Marly.43

Poiché Rossi aveva nominato Quadri come il primo dei suoi allievi, e poiché, come abbiamo visto, lo stesso Quadri era in Russia a partire dal 1700 circa e nei documenti della Cancelleria per l'edilizia di San

42 RGIA, busta 51a, ff. 82-100; Ibidem, fondo 470 , inv. 5, busta 21 , ff. 5,6 (“ $\ldots$ во оном мастере имеетца нужда в Питер гоф в Стрелиной мызе також и в Санкт Питербурхе святой церкве Петра и Павла и в других местах для штукатурных работ...").

43 Ibidem, fondo 467, Inv. 4, busta 810, f. 8 . 
Pietroburgo figura come inviato da $\mathrm{Mosca}^{44}$, non sussiste più alcun dubbio sul fatto che egli lavorò nella bottega moscovita di Rossi.

Un altro collaboratore pluriennale di Rossi fu Pietro Antonio Gielpi, che abbiamo già menzionato, il quale giunse a Mosca con Trezzini nel 1703. Egli si distingue dai compagni di viaggio per essere "maestro nei lavori in gesso". ${ }^{45}$ Per questa ragione è probabile che Rossi non lo citi tra i suoi allievi. Arrivato a Mosca, Gielpi presentò nel 1704 una richiesta di rimborso per le spese di viaggio. In quel documento egli figura come "lo straniero, maestro nei lavori di taglio e nei lavori figurativi in pietra, Petr Antonov". 46

Si può supporre che Gielpi riuscè a concretizzare il proprio talento a Mosca ed a unirsi all'atelier di Rossi, nel quale avrebbe lavorato per un lungo periodo. All'inizio del 1718 Gielpi e Rossi rassegnarono le loro dimissioni a Pietro I. Evidentemente Gielpi e Rossi lasciarono San Pietroburgo insieme. Sebbene non disponiamo di dati precisi sulle origini di Gielpi, possiamo ipotizzare che anch'egli provenisse dalla Svizzera italiana. Il nome di Gielpi non compare più tardi nei documenti russi. Rossi, invece, tornò a Pietroburgo insieme con i figli. Negli anni 1722-1723 lavorò come mastro privato alla costruzione del cosiddetto Palazzo Italiano sulle rive della Fontanka, destinato per la zarina Caterina. Nel marzo 1724 egli ritornò al servizio della Cancelleria per l'edilizia, ma morì a Pietroburgo nel gennaio $1725^{47}$

I legami degli artisti ticinesi con la Russia nel primo quarto del XVIII secolo furono dunque più stretti di quanto si è ritenuto in passato.

44 RGIA, fondo 467 , Inv. 4, busta 118 , f. 475 .

45 RGADA, fondo 150, anno 1703, busta 12, f. 21 verso (“гипсового дела мастер”).

46 Ibidem, fondo 141 , inv. 8, anno 1704 busta 55 , f. 3.

47 Per l'attività di Giovanni Francesco Rossi in Russia vedi anche: Sergey O. Androsov, Russkie zakazčiki i italianskie chudožniki v XVIII veke (Committenti russi e Artisti italiani ne secolo XVIII) (San Pietroburgo: D. Bulanin, 2003), 52-77.

\section{APPENDICE DOCUMENTARIA. IL CONTRATTO DI DOMENICO TREZZINI ${ }^{48}$}

In Nomine Domini Copenhaghen il Primo D'aprile 1703

Io Andrea Petrovich Ismaelovf Ambasciatore extraordinario quà alla Corte Di Sua Maesta di Denimarcha o Fatto la Conventione che segue a nome et ordine Di Sua Maesta Cexarea Grand Duca Di Moscovia Mio Padrone, Prometto a Domenico Trezzino Capo Mastro Italiano Di Natione il quale o trovato quà nel Servitio Di Sua Maesta Di Denimarca lasciando quà li suoi impieghi et servitio per andare in Moscovia per servire a d[ett]a Maesta Cexarea Mio Padrone il qualità di capo Mastro di Ba[timenti] Fabriche et Forificationi in riguardo della sua Abilità e servitio li Prometto No[me Di] Sua Maesta Cexarea per sua Mercede vinti ongari d'oro per ciascun Mese sintende tutto... et Cominciarà a Correre il suo Stipendio il di Primo Aprile et sarà Pagato tutti li Mese in boni dinari corenti che siano valuta tanto nel Paese quanto F[u]ori del Paese cioè l'ongaro che sia la valuta di sette Marchi libse per ciascuno Corenti in Denimarcha come ancora il sud(ett)o Trezzino facendo vedere la sua abilità li Prometto ancho di Fare li argumentare di salario sarà ancho permesso che Presentando l'ocasione di Fare qualche intrapresa Di Fabriche buero (?) di Fortificatione che il d[ett] o Trezzino possa interpren[dere] e che corrà il continuamente il suo solario come ancora se qualche Particolare le Comandasse o per Fare Discorsi o per inspechtione di Fabriche li sarà permesso et lo sicuriamo ancho il d[ett]o Trezzino che non volendo più servire o che l'aria non le conferisse o per ritornare al suo Paese o volendo Partire per altri Paesi che di subito li sarà data la sua licenza di partire del Paese senza alcuna contrarietà e molestia anzi Facilità di condurlo et Raccomandarlo d'un loco à l'altro nel Paese... ancora al sud[ett]o li sarà Parlato per il suo viagio di andare in Moscovia scudi cinquanta di moneta di Denimarch senza essere compresi nel Conto come ancora quando il sud[ett]o vorrà Partire che siano tenuti a sborsagli il Medemo Dinaro come ancora, che il sud[ett]o possa portar seco la poca Facoltà che aveva Profittato d[ett]o Paese, et se il sud[ett]o per disgratia cadesse amalato per qualche tempo che il stipendio li Continua et ocorendo che il sud[ett]o avesse ocasione di viagiare per qualche Parte per servitio di Sua Maesta Cexarea che li siano per abate (?) et in Fede siano sottascritti di Proprio Pugno con il n[ost]ro Sigillo.

Confeso Io Domenicho Trezino di avere Ricevute di sua Ecelenza Ambasciatore schudi conquanta di oro 50 per Fare il mio viaggio.

48 RGADA, fondo 150 , anno 1703 , busta 12 , f. 51 . 
Sergey Androsov: Domenico Trezzini and other Ticinese masters at Saint Petersburg and Tallinn from the beginning OF THE 18TH CENTURY

Keywords: Domenico Trezzini; Ticinese masters; Saint Petersburg; Tallinn; notes on Italian architects; 18th Century

\section{CV}

Sergey Androsov (b. 1948), PhD has been the Head of the Department of European Figurative Art at the State Hermitage Museum since 2006. He is the author of more than 350 scientific publications on Italian and Russian art from the 14th to 20th centuries. Androsov is also a member of Ateneo Veneto (Italy) and the Academy of Natural Science (Russia).

\section{SUMMARY}

The activities and work of architect Domenico Trezzini (1670-1734), and the masters that arrived in Russia with him, have been researched for a long time, and many contentions have been concretised or disproven at various times. This article focuses on the issue of how masters from a single area - Ticino, Switzerland - ended up creating important fortifications, palaces and churches in the Russian Empire during the reign of Peter the Great at the beginning of 18th century. The author describes the movement of Trezzini and the Ticino masters from Copenhagen to Moscow as reported in various historical sources and the works of Danish and Russian researchers. 